\title{
Carleson inequalities on parabolic Hardy spaces
}

\author{
Hayato NAKAgAWA and Noriaki SuzUKI
}

(Received July 14, 2014; Revised September 30, 2014)

\begin{abstract}
We study Carleson inequalities in a framework of parabolic Hardy spaces. Similar results for parabolic Bergman spaces are discussed in [NSY1] (see also [NSY2]), where $\tau$-Carleson measures play an important roll. In the present case, $T_{\tau}$-Carleson measures are useful. We give an relation between these measures.
\end{abstract}

Key words: Carleson inequality, parabolic operator, Hardy space, Carleson measure.

\section{Introduction}

For an integer $n \geq 1$, let $\mathbb{R}_{+}^{n+1}:=\left\{(x, t) \in \mathbb{R}^{n+1} \mid x=\left(x_{1}, x_{2}, \ldots, x_{n}\right) \in\right.$ $\left.\mathbb{R}^{n}, t>0\right\}$ denote the upper half space. For $0<\alpha \leq 1$, let $L^{(\alpha)}$ be a parabolic operator

$$
L^{(\alpha)}:=\partial_{t}+\left(-\Delta_{x}\right)^{\alpha}, \quad \Delta_{x}:=\frac{\partial^{2}}{\partial x_{1}^{2}}+\frac{\partial^{2}}{\partial x_{2}^{2}}+\cdots+\frac{\partial^{2}}{\partial x_{n}^{2}} .
$$

We say that a continuous function $u$ on $\mathbb{R}_{+}^{n+1}$ is an $L^{(\alpha)}$-harmonic function if $L^{(\alpha)} u=0$ in the sense of distributions, which is defined later.

For $1<p<\infty$, we denote by $h_{\alpha}^{p}:=h_{\alpha}^{p}\left(\mathbb{R}_{+}^{n+1}\right)$ the set of all $L^{(\alpha)}{ }_{-}$ harmonic functions with $\|u\|_{h_{\alpha}^{p}}<\infty$, where

$$
\|u\|_{h_{\alpha}^{p}}:=\sup _{t>0}\left(\int_{\mathbb{R}^{n}}|u(x, t)|^{p} d x\right)^{1 / p} .
$$

It is shown that $h_{\alpha}^{p}$ is a Banach space under the norm $\|\cdot\|_{h_{\alpha}^{p}}$ (see Section 2). We call $h_{\alpha}^{p}$ the $\alpha$-parabolic Hardy space of order $p$.

Let $1<p<\infty$ and $1<q<\infty$. We say that a positive Borel measure $\mu$ on $\mathbb{R}_{+}^{n+1}$ satisfies a $(p, q)$-Carleson inequality on parabolic Hardy spaces if the inclusion mapping from $h_{\alpha}^{p}$ to $L^{q}\left(\mathbb{R}_{+}^{n+1}, d \mu\right)$ is bounded, that is, there exists a positive constant $C$ such that 


$$
\|u\|_{L^{q}\left(\mathbb{R}_{+}^{n+1}, d \mu\right)} \leq C\|u\|_{h_{\alpha}^{p}}
$$

holds for all $u \in h_{\alpha}^{p}$. To study (1), the following definition is useful.

Definition 1 Let $\mu$ be a positive Borel measure on $\mathbb{R}_{+}^{n+1}$ and $\tau$ be a positive number. We say that $\mu$ is a $T_{\tau}$-Carleson measure if there exists a positive constant $C$ such that

$$
\mu\left(T^{(\alpha)}(x, t)\right) \leq C t^{(n / 2 \alpha) \tau}
$$

holds for all $(x, t) \in \mathbb{R}_{+}^{n+1}$, where

$$
T^{(\alpha)}(x, t):=\left\{(y, s) \in \mathbb{R}_{+}^{n+1}|| y-\left.x\right|^{2 \alpha}+s \leq t\right\} .
$$

We are now ready to state our main theorem.

Theorem 1 Let $1<p \leq q<\infty$ and $\mu$ be a positive Borel measure on $\mathbb{R}_{+}^{n+1}$. Then $\mu$ satisfies a $(p, q)$-Carleson inequality if and only if $\mu$ is a $T_{q / p}$-Carleson measure.

A Carleson inequality on parabolic Bergman spaces is already proved in [NSY1] (see also [NSY2]). We discuss a relation between two inequalities in Section 4. As a result, we will see that a positive Borel measure $\mu$ satisfies a $(p, q)$-Carleson inequality on parabolic Hardy spaces if and only if $\mu$ satisfies a $\left(p^{\prime}, q^{\prime}\right)$-Carleson inequality on parabolic Bergman spaces, where $(q / p)(n / 2 \alpha)=\left(q^{\prime} / p^{\prime}\right)(n / 2 \alpha+1)$ (see Corollary 1 below).

Throughout the paper, we will use the same letter $C$ to denote various positive constants; it may vary even within a line.

\section{Preliminaries}

In order to define an $L^{(\alpha)}$-harmonic function, we recall how the adjoint operator $\widetilde{L}^{(\alpha)}=-\partial_{t}+\left(-\Delta_{x}\right)^{\alpha}$ acts on $C_{c}^{\infty}\left(\mathbb{R}_{+}^{n+1}\right)$, where $C_{c}^{\infty}\left(\mathbb{R}_{+}^{n+1}\right)$ is the set of all $C^{\infty}$-functions with compact support on $\mathbb{R}_{+}^{n+1}$. Since it is trivial when $\alpha=1$, we only consider for $0<\alpha<1$ here. Then $\left(-\Delta_{x}\right)^{\alpha}$ is the convolution operator defined by $-c_{n, \alpha}$ p.f. $|x|^{-n-2 \alpha}$, where

$$
c_{n, \alpha}=4^{\alpha} \pi^{-n / 2} \Gamma((2 n+\alpha) / 2) /|\Gamma(-\alpha)|, \quad|x|=\left(x_{1}^{2}+x_{2}^{2}+\cdots+x_{n}^{2}\right)^{1 / 2},
$$


and $\Gamma(\cdot)$ is the gamma function. Hence for $\varphi \in C_{c}^{\infty}\left(\mathbb{R}_{+}^{n+1}\right)$,

$$
\widetilde{L}^{(\alpha)} \varphi(x, t)=-\frac{\partial}{\partial t} \varphi(x, t)-c_{n, \alpha} \lim _{\delta \downarrow 0} \int_{|y|>\delta}(\varphi(x+y, t)-\varphi(x, t))|y|^{-n-2 \alpha} d y .
$$

A function $h$ on $\mathbb{R}_{+}^{n+1}$ is said to be $L^{(\alpha)}$-harmonic if $h$ is continuous,

$$
\int_{t_{1}}^{t_{2}} \int_{\mathbb{R}^{n}}|h(x, t)|(1+|x|)^{-n-2 \alpha} d x d t<\infty
$$

for every $0<t_{1}<t_{2}<\infty$ and $\iint_{\mathbb{R}_{+}^{n+1}} h \cdot \widetilde{L}^{(\alpha)} \varphi d x d t=0$ holds for all $\varphi \in C_{c}^{\infty}\left(\mathbb{R}_{+}^{n+1}\right)$. Note that the condition (4) is equivalent to $\iint_{\mathbb{R}_{+}^{n+1}} \mid h$. $\widetilde{L}^{(\alpha)} \varphi \mid d x d t<\infty$ for all $\varphi \in C_{c}^{\infty}\left(\mathbb{R}_{+}^{n+1}\right)$ (see [NSS1]).

We use the fundamental solution $W^{(\alpha)}$ of $L^{(\alpha)}$, which is defined by

$$
W^{(\alpha)}(x, t)= \begin{cases}(2 \pi)^{-n} \int_{\mathbb{R}^{n}} e^{-t|\xi|^{2 \alpha}} e^{i x \cdot \xi} d \xi & t>0 \\ 0 & t \leq 0\end{cases}
$$

where $x \cdot \xi$ is the inner product of $x$ and $\xi$. It is known that when $\alpha=1 / 2$, $W^{(1 / 2)}$ coincides with the Poisson kernel on $\mathbb{R}_{+}^{n+1}$, that is, for $t>0$,

$$
W^{(1 / 2)}(x, t)=\frac{\Gamma\left(\frac{n+1}{2}\right)}{\pi^{(n+1) / 2}} \frac{t}{\left(|x|^{2}+t^{2}\right)^{(n+1) / 2}} .
$$

Note also that $W^{(1)}(x, t)=(4 \pi t)^{-n / 2} e^{-|x|^{2} / 4 t}$ is the Gauss kernel. We recall some properties of the fundamental solution (see [NSS1] and [NSS2] for details).

For any compact set $K$ in $\mathbb{R}_{+}^{n+1}$, there exists a positive constant $C$ such that

$$
\inf _{(x, t) \in K} W^{(\alpha)}(x, t)>C
$$

For every positive $t$,

$$
\int_{\mathbb{R}^{n}} W^{(\alpha)}(x, t) d x=1
$$


and for any positive $s, t$ with $0<s<t$,

$$
W^{(\alpha)}(x, t)=\int_{\mathbb{R}^{n}} W^{(\alpha)}(x-y, t-s) W^{(\alpha)}(y, s) d y .
$$

By a change of variables, we see

$$
W^{(\alpha)}(x, t)=t^{-n / 2 \alpha} W^{(\alpha)}\left(t^{-1 / 2 \alpha} x, 1\right) .
$$

The following estimate is useful: there exists a constant $C>0$ such that

$$
W^{(\alpha)}(x, t) \leq C \frac{t}{\left(t+|x|^{2 \alpha}\right)^{n / 2 \alpha+1}} .
$$

It is known that the usual harmonic Hardy space $H^{p}$ on the upper half space is naturally equivalent with the space $L^{p}\left(\mathbb{R}^{n}\right)$. The same identity also holds in our case. For $f \in L^{p}\left(\mathbb{R}^{n}\right)$, we set

$$
P^{(\alpha)}[f](x, t):=\int_{\mathbb{R}^{n}} W^{(\alpha)}(x-y, t) f(y) d y .
$$

Then we have the following proposition.

Proposition 1 Let $1<p<\infty$. For each $u \in h_{\alpha}^{p}$, there exists a unique function $f \in L^{p}\left(\mathbb{R}^{n}\right)$ such that $u=P^{(\alpha)}[f]$. Conversely, for any $f \in L^{p}\left(\mathbb{R}^{n}\right), P^{(\alpha)}[f] \in h_{\alpha}^{p}$. Moreover, $\left\|P^{(\alpha)}[f]\right\|_{h_{\alpha}^{p}}=\|f\|_{L^{p}\left(\mathbb{R}^{n}\right)}$ holds.

Proof. By (7), (8) and (10), the assertion follows from a quite similar proof to the usual harmonic Hardy space (cf. [S, p. 62 and p. 200]). Here we only check that $P^{(\alpha)}[f]$ is $L^{(\alpha)}$-harmonic when $f \in L^{p}\left(\mathbb{R}^{n}\right)$. Let $q$ be such as $1 / p+1 / q=1$. For $0<t_{1}<t_{2}<\infty$, by (10) and the Hölder inequality, we have

$$
\begin{aligned}
\int_{t_{1}}^{t_{2}} & \int_{\mathbb{R}^{n}}\left|P^{(\alpha)}[f](x, t)\right|(1+|x|)^{-n-2 \alpha} d x d t \\
\leq & \int_{t_{1}}^{t_{2}} \int_{\mathbb{R}^{n}}\left(\int_{\mathbb{R}^{n}}\left(W^{(\alpha)}(x-y, t)\right)^{q} d y\right)^{1 / q} \\
& \times\|f\|_{L^{p}\left(\mathbb{R}^{n}\right)}(1+|x|)^{-n-2 \alpha} d x d t
\end{aligned}
$$




$$
\begin{aligned}
\leq & C \int_{t_{1}}^{t_{2}} \int_{\mathbb{R}^{n}}\left(\int_{\mathbb{R}^{n}} \frac{t^{q}}{\left(|x-y|^{2 \alpha}+t\right)^{(n / 2 \alpha+1) q}} d y\right)^{1 / q} \\
& \times\|f\|_{L^{p}\left(\mathbb{R}^{n}\right)}(1+|x|)^{-n-2 \alpha} d x d t \\
\leq & C\left(\frac{\omega_{n-1}}{2 \alpha} \int_{0}^{\infty} \zeta^{n / 2 \alpha-1}(1+\zeta)^{-(n / 2 \alpha+1) q} d \zeta\right)^{1 / q} \\
& \times\|f\|_{L^{p}\left(\mathbb{R}^{n}\right)} \int_{t_{1}}^{t_{2}} t^{-(n / 2 \alpha)(1 / p)} d t \int_{\mathbb{R}^{n}}(1+|x|)^{-n-2 \alpha} d x \\
<\infty &
\end{aligned}
$$

where $\omega_{n-1}$ is the surface area of unit sphere in $\mathbb{R}^{n}$. Hence by the Fubini theorem,

$$
\begin{aligned}
& \iint_{\mathbb{R}_{+}^{n+1}} P^{(\alpha)}[f](x, t) \cdot \widetilde{L}^{(\alpha)} \varphi(x, t) d x d t \\
& \quad=\iint_{\mathbb{R}_{+}^{n+1}}\left(\int_{\mathbb{R}^{n}} W^{(\alpha)}(x-y, t) f(y) d y\right) \widetilde{L}^{(\alpha)} \varphi(x, t) d x d t \\
& \quad=\iint_{\mathbb{R}^{n}}\left(\iint_{\mathbb{R}_{+}^{n+1}} W^{(\alpha)}(x-y, t) \widetilde{L}^{(\alpha)} \varphi(x, t) d x d t\right) f(y) d y \\
& =0
\end{aligned}
$$

because the fundamental solution is $L^{(\alpha)}$-harmonic.

When $u=P_{\alpha}[f]$, then $|u(x, t)| \leq\|W(x-\cdot, t)\|_{L^{q}\left(\mathbb{R}^{n}\right)}\|f\|_{L^{p}\left(\mathbb{R}^{n}\right)}$ holds for $1 / p+1 / q=1$. This shows that a Cauchy sequence $\left\{u_{n}\right\}$ in $h_{\alpha}^{p}$ implies compact uniform convergence of $\left\{u_{n}\right\}$, so that $h_{\alpha}^{p}$ forms a Banach space. This proposition also shows that $h_{1 / 2}^{p}$ is just the usual harmonic Hardy space $H^{p}$.

\section{Proof of Theorem 1}

In this section, we will give a proof of Theorem 1. The "only if" part is not difficult. It follows from direct computations of integrals of $W^{(\alpha)}$. Let $1<p<\infty, 1<q<\infty$ and suppose that $\mu$ satisfies a Carleson inequality (1). We fix $(x, t) \in \mathbb{R}_{+}^{n+1}$ and put $u(y, s)=W^{(\alpha)}(x-y, t+s)$. Then $u \in h_{\alpha}^{p}$. 
In fact, by (10),

$$
\begin{aligned}
\|u\|_{h_{\alpha}^{p}}^{p} & =\sup _{s>0} \int_{\mathbb{R}^{n}} W^{(\alpha)}(x-y, t+s)^{p} d y \\
& \leq C \sup _{s>0} \int_{\mathbb{R}^{n}}\left(\frac{t+s}{\left(t+s+|x-y|^{2 \alpha}\right)^{n / 2 \alpha+1}}\right)^{p} d y \\
& =C \frac{\omega_{n-1}}{2 \alpha} \sup _{s>0}(t+s)^{(n / 2 \alpha)(1-p)} \int_{0}^{\infty} \frac{\eta^{n / 2 \alpha-1}}{(1+\eta)^{(n / 2 \alpha+1) p}} d \eta \\
& \leq C t^{(n / 2 \alpha)(1-p)}
\end{aligned}
$$

On the other hand, since $|x-y|^{2 \alpha} \leq t-s<t+s$ for every $(y, s) \in T^{(\alpha)}(x, t)$, (6) and (9) show

$u(y, s)=(t+s)^{-n / 2 \alpha} W^{(\alpha)}\left(\frac{x-y}{(t+s)^{1 / 2 \alpha}}, 1\right) \geq C(t+s)^{-n / 2 \alpha} \geq C(2 t)^{-n / 2 \alpha}$

with some constant $C>0$. This implies that

$$
\|u\|_{L^{q}\left(\mathbb{R}_{+}^{n+1}, d \mu\right)}^{q} \geq \iint_{T^{(\alpha)}(x, t)} u(y, s)^{q} d \mu(y, s) \geq C t^{-(n / 2 \alpha) q} \mu\left(T^{(\alpha)}(x, t)\right) .
$$

Hence the inequality (1) gives us

$$
t^{-n / 2 \alpha} \mu\left(T^{(\alpha)}(x, t)\right)^{1 / q} \leq C\|u\|_{L^{q}\left(\mathbb{R}_{+}^{n+1}, d \mu\right)} \leq C\|u\|_{h_{\alpha}^{p}} \leq C t^{(n / 2 \alpha)(1 / p-1)},
$$

which shows $\mu$ is a $T_{q / p}$-Carleson measure. Here we remark that we do not assume $p \leq q$ in the above argument.

To show the "if" part, we use a Luecking's idea (see [L]). In the sequel, we denote by $B(x, r)$ the ball with center $x$ and radius $r$ in the boundary of upper half space, that is $B(x, r)=\left\{y \in \mathbb{R}^{n}|| x-y \mid<r\right\}$. For an open set $E$ in $\mathbb{R}^{n}$, we set

$$
\widehat{E}:=\left\{(x, t) \in \mathbb{R}_{+}^{n+1} \mid B\left(x, t^{1 / 2 \alpha}\right) \subseteq E\right\}
$$

Let $(x, t) \in \mathbb{R}_{+}^{n+1}$. When $\alpha \leq 1 / 2$, then $(t-s)^{1 / 2 \alpha} \leq t^{1 / 2 \alpha}-s^{1 / 2 \alpha} \leq$ $(2 t-s)^{1 / 2 \alpha}$ holds for $0<s \leq t$. Hence $T^{(\alpha)}(x, t) \subset B\left(\widehat{x, t^{1 / 2} \alpha}\right) \subset T^{(\alpha)}(x, 2 t)$. 
When $\alpha>1 / 2$, then $t^{1 / 2 \alpha}-s^{1 / 2 \alpha} \leq(t-s)^{1 / 2 \alpha} \leq(2 t)^{1 / 2 \alpha}-s^{1 / 2 \alpha}$ holds for $0<s \leq t$, and hence $B\left(\widehat{x, t^{1 / 2}} \alpha\right) \subset T^{(\alpha)}(x, t) \subset B\left(x, \widehat{(2 t)^{1}} / 2 \alpha\right)$. Therefore

$$
B\left(x{\widehat{(t / 2)^{1}}}^{1 / 2 \alpha}\right) \subset T^{(\alpha)}(x, t) \subset B\left(x \widehat{{(2 t)^{1}}^{2}}{ }^{\alpha}\right)
$$

holds for all $0<\alpha \leq 1$.

Let $1<p<\infty$. We also use the maximal function $M f$, which is defined by

$$
M f(x):=\sup _{r>0} \frac{1}{r^{n}} \int_{B(x, r)}|f(y)| d y .
$$

for $f \in L^{p}\left(\mathbb{R}^{n}\right)$. It is known that

$$
\|M f\|_{L^{p}\left(\mathbb{R}^{n}\right)} \leq C_{p, n}\|f\|_{L^{p}\left(\mathbb{R}^{n}\right)}
$$

where $C_{p, n}=2\left(5^{n} p /(p-1)\right)^{1 / p}$ (see $\left.[\mathrm{S}, \mathrm{p} .5]\right)$.

Now we return to the proof of the "if" part. Assume that $1<p \leq q<\infty$ and $\mu$ is a $T_{q / p}$-Carleson measure. Then by (13),

$$
\mu\left(B\left(\widehat{x, t^{1 / 2}} \alpha\right)\right) \leq C t^{(n / 2 \alpha)(q / p)}
$$

with some constant $C>0$. We use the following notations. For $u \in h_{\alpha}^{p}$ and $x \in \mathbb{R}^{n}$, we set

$$
u^{*}(x):=\sup _{(y, s) \in \Omega(x)}|u(y, s)|
$$

where $\Omega(x):=\left\{(y, s) \in \mathbb{R}_{+}^{n+1}|| y-x \mid<s^{1 / 2 \alpha}\right\}$ and for $\lambda>0$, we set

$$
\begin{aligned}
& E_{\lambda}:=\left\{x \in \mathbb{R}^{n} \mid u^{*}(x)>\lambda\right\}, \\
& G_{\lambda}:=\left\{(x, t) \in \mathbb{R}_{+}^{n+1}|| u(x, t) \mid>\lambda\right\} .
\end{aligned}
$$

Let $\left(x_{0}, t_{0}\right) \in G_{\lambda}$ and take any $z \in B\left(x_{0}, t_{0}^{1 / 2 \alpha}\right)$. Since $\left(x_{0}, t_{0}\right) \in \Omega(z)$, we have $u^{*}(z)>\lambda$, and hence $B\left(x_{0}, t_{0}^{1 / 2 \alpha}\right) \subset E_{\lambda}$. This shows

$$
G_{\lambda} \subset \widehat{E_{\lambda}}
$$


For subsets $X$ and $Y$ in $\mathbb{R}^{n}$, we denote by $\partial X$ the boundary of $X$, by $\operatorname{diam}(X)$ the diameter of $X$ and by $\operatorname{dist}(X, Y)$ the distance between $X$ and $Y$. Since $u^{*}$ is lower semicontinuous, $E_{\lambda}$ is an open set. Hence we have the following Whitney decomposition;

$$
E_{\lambda}=\bigcup_{k=1}^{\infty} Q_{k}
$$

where $\left\{Q_{k}\right\}$ are closed cubes whose sides are parallel to the axes and whose interiors are mutually disjoint, and satisfy

$$
\operatorname{diam}\left(Q_{k}\right) \leq \operatorname{dist}\left(Q_{k}, \partial E_{\lambda}\right) \leq 4 \operatorname{diam}\left(Q_{k}\right)
$$

(see $[\mathrm{S}, \mathrm{p} .16])$. Then there exists a constant $C>0$ such that

$$
\widehat{E_{\lambda}} \subset \bigcup_{k=1}^{\infty} \widehat{C Q_{k}}
$$

where $C Q_{k}$ is a cube with $C$ times diameter and the common center as $Q_{k}$. In fact, take $(x, t) \in \widehat{E_{\lambda}}$. Since $B\left(x, t^{1 / 2 \alpha}\right) \subset E_{\lambda}$, we choose $t_{0} \geq t$ such that $\operatorname{dist}\left(B\left(x, t_{0}^{1 / 2 \alpha}\right), \partial E_{\lambda}\right)=0$. By (18), $x \in Q_{k_{0}}$ for some integer $k_{0} \geq 1$. Let $x_{0}$ be the center of $Q_{k_{0}}$ and $\widetilde{x}_{0}$ be a point in $Q_{k_{0}}$ such that $\operatorname{dist}\left(\widetilde{x}_{0}, \partial E_{\lambda}\right)=\operatorname{dist}\left(Q_{k_{0}}, \partial E_{\lambda}\right)$. Then for any $y \in B\left(x, t_{0}^{1 / 2 \alpha}\right)$, we have

$$
\begin{aligned}
\left|y-x_{0}\right| & \leq|y-x|+\left|x-x_{0}\right| \\
& \leq t_{0}^{1 / 2 \alpha}+\operatorname{diam}\left(Q_{k_{0}}\right) \\
& \leq\left|x-\widetilde{x}_{0}\right|+\operatorname{dist}\left(Q_{k_{0}}, \partial E_{\lambda}\right)+\operatorname{diam}\left(Q_{k_{0}}\right) \\
& \leq \operatorname{diam}\left(Q_{k_{0}}\right)+4 \operatorname{diam}\left(Q_{k_{0}}\right)+\operatorname{diam}\left(Q_{k_{0}}\right) \\
& =6 \operatorname{diam}\left(Q_{k_{0}}\right) .
\end{aligned}
$$

This shows that $y \in C Q_{k_{0}}$, where $C=12 \sqrt{n}$. Since $y$ is an arbitary point in $B\left(x, t_{0}^{1 / 2 \alpha}\right)$, we have $B\left(x, t^{1 / 2 \alpha}\right) \subset B\left(x, t_{0}^{1 / 2 \alpha}\right) \subset C Q_{k_{0}}$, and hence $(x, t) \in$ $\widehat{C Q_{k_{0}}}$. This shows (19).

Next we estimate the $L^{p}$ norm of $u^{*}$. There exists a positive constant $C$ such that for every $u \in h_{\alpha}^{p}$, 


$$
\left\|u^{*}\right\|_{L^{p}\left(\mathbb{R}^{n}\right)} \leq C\|u\|_{h_{\alpha}^{p}}
$$

In fact, as in Proposition 1 , we take $f \in L^{p}\left(\mathbb{R}^{n}\right)$ such that $u=P^{(\alpha)}[f]$ and let $x \in \mathbb{R}^{n}$. Take $(y, s) \in \Omega(x)$ and $z \in \mathbb{R}^{n}$ arbitrarily. Then

$$
\begin{aligned}
s+|x-z|^{2 \alpha} & \leq s+(|x-y|+|y-z|)^{2 \alpha} \leq s+\left(s^{1 / 2 \alpha}+|y-z|\right)^{2 \alpha} \\
& \leq\left(2^{2 \alpha}+1\right)\left(s+|y-z|^{2 \alpha}\right) .
\end{aligned}
$$

Hence by (5), we have

$$
\begin{aligned}
|u(y, s)| & \leq \int_{\mathbb{R}^{n}}|f(z)| W^{(\alpha)}(y-z, s) d z \\
& \leq C \int_{\mathbb{R}^{n}} \frac{s|f(z)|}{\left(s+|y-z|^{2 \alpha}\right)^{n / 2 \alpha+1}} d z \\
& \leq C \int_{\mathbb{R}^{n}} \frac{s|f(z)|}{\left(s+|x-z|^{2 \alpha}\right)^{n / 2 \alpha+1}} d z \\
& =C \sum_{m=0}^{\infty} I_{m},
\end{aligned}
$$

where

$$
\begin{aligned}
& I_{0}:=\int_{|x-z|<s^{1 / 2 \alpha}} \frac{s|f(z)|}{\left(s+|x-z|^{2 \alpha}\right)^{n / 2 \alpha+1}} d z \\
& I_{m}:=\int_{\left(2^{m-1} s\right)^{1 / 2 \alpha} \leq|x-z|<\left(2^{m} s\right)^{1 / 2 \alpha}} \frac{s|f(z)|}{\left(s+|x-z|^{2 \alpha}\right)^{n / 2 \alpha+1}} d z \\
& \quad(m=1,2, \ldots) .
\end{aligned}
$$

Then

$$
I_{0} \leq \int_{|x-z|<s^{1 / 2 \alpha}} \frac{s|f(z)|}{s^{n / 2 \alpha+1}} d z=s^{-n / 2 \alpha} \int_{|x-z|<s^{1 / 2 \alpha}}|f(z)| d z \leq M f(x)
$$

and 


$$
\begin{aligned}
I_{m} & =\int_{\left(2^{m-1} s\right)^{1 / 2 \alpha} \leq|x-z|<\left(2^{m} s\right)^{1 / 2 \alpha}} \frac{s|f(z)|}{\left(s+2^{m-1} s\right)^{n / 2 \alpha+1}} d z \\
& \leq \frac{1}{\left(1+2^{m-1}\right)^{n / 2 \alpha+1}} \frac{1}{s^{n / 2 \alpha}} \int_{|x-z|<\left(2^{m} s\right)^{1 / 2 \alpha}}|f(z)| d z \\
& \leq 2^{-m} 2^{(n / 2 \alpha+1)}\left(2^{m} s\right)^{-n / 2 \alpha} \int_{|x-z|<\left(2^{m} s\right)^{1 / 2 \alpha}}|f(z)| d z \\
& \leq 2^{-m} 2^{(n / 2 \alpha+1)} \operatorname{Mf}(x)
\end{aligned}
$$

implies

$$
\sum_{m=1}^{\infty} I_{m} \leq 2^{(n / 2 \alpha+1)} M f(x)
$$

and hence $|u(y, s)| \leq C M f(x)$ holds. Since $(y, s) \in \Omega(x)$ is arbitrary, we have $u^{*}(x) \leq C M f(x)$ for all $x \in \mathbb{R}^{n}$. This and (14) show (20).

Now we will finish the proof of the "if" part. By (15), we see $\mu(\widehat{Q}) \leq$ $C|Q|^{q / p}$ for every cube $Q$, and hence (17), (18) and (19) show

$$
\mu\left(G_{\lambda}\right) \leq \mu\left(\widehat{E_{\lambda}}\right) \leq \sum_{k=1}^{\infty} \mu\left(\widehat{C Q_{k}}\right) \leq C \sum_{k=1}^{\infty}\left|Q_{k}\right|^{q / p} \leq C\left|E_{\lambda}\right|^{q / p}
$$

because $q / p \geq 1$, where $|G|$ denotes the volume of a Borel set $G$ in $\mathbb{R}^{n}$. Then by $(21)$

$$
\begin{aligned}
\|u\|_{L^{q}\left(\mathbb{R}_{+}^{n+1}, d \mu\right)}^{q} & =\iint_{\mathbb{R}_{+}^{n+1}}|u(x, t)|^{q} d \mu(x, t)=q \int_{0}^{\infty} \mu\left(G_{\lambda}\right) \lambda^{q-1} d \lambda \\
& \leq C \int_{0}^{\infty}\left|E_{\lambda}\right|^{q / p} \lambda^{q-1} d \lambda=C \sum_{k=-\infty}^{\infty} \int_{2^{k}}^{2^{k+1}}\left|E_{\lambda}\right|^{q / p} \lambda^{q-1} d \lambda \\
& \leq C \sum_{k=-\infty}^{\infty} \int_{2^{k}}^{2^{k+1}}\left|E_{2^{k}}\right|^{q / p} 2^{(k+1)(q-1)} d \lambda \\
& \leq C \sum_{k=-\infty}^{\infty} 2^{(k+1) q}\left|E_{2^{k}}\right|^{q / p} .
\end{aligned}
$$


On the other hand,

$$
\begin{aligned}
\left\|u^{*}\right\|_{L^{p}\left(\mathbb{R}^{n}\right)}^{p} & =\int_{\mathbb{R}^{n}}\left|u^{*}(x)\right|^{p} d x=p \int_{0}^{\infty}\left|E_{\lambda}\right| \lambda^{p-1} d \lambda \\
& \geq p \sum_{k=-\infty}^{\infty} \int_{2^{k-1}}^{2^{k}}\left|E_{2^{k}}\right| 2^{(k-1)(p-1)} d \lambda \\
& =p \sum_{k=-\infty}^{\infty} 2^{(k-1) p}\left|E_{2^{k}}\right|=\frac{p}{2^{2 p}} \sum_{k=-\infty}^{\infty} 2^{(k+1) p}\left|E_{2^{k}}\right| .
\end{aligned}
$$

Since $p \leq q$, we have

$$
\begin{aligned}
\|u\|_{L^{q}\left(\mathbb{R}_{+}^{n+1}, d \mu\right)}^{p} & \leq C\left(\sum_{k=-\infty}^{\infty} 2^{(k+1) q}\left|E_{2^{k}}\right|^{q / p}\right)^{p / q} \\
& \leq C \sum_{k=-\infty}^{\infty} 2^{(k+1) p}\left|E_{2^{k}}\right| \leq C\left\|u^{*}\right\|_{L^{p}\left(\mathbb{R}^{n}\right)}^{p} .
\end{aligned}
$$

This together with (20) gives us the Carleson inequality (1). This completes the proof.

\section{A relation between $T_{\tau}$-Carleson measures and $\tau$-Carleson measures}

We recall a result for parabolic Bergman spaces. For $1 \leq p<\infty$, we denote by $b_{\alpha}^{p}:=b_{\alpha}^{p}\left(\mathbb{R}_{+}^{n+1}\right)$ the set of all $L^{(\alpha)}$-harmonic functions $u$ with $\|u\|_{L^{p}\left(\mathbb{R}_{+}^{n+1}\right)}<\infty$, where

$$
\|u\|_{L^{p}\left(\mathbb{R}_{+}^{n+1}\right)}:=\left(\iint_{\mathbb{R}_{+}^{n+1}}|u(x, t)|^{p} d x d t\right)^{1 / p} .
$$

We call $b_{\alpha}^{p}$ the $\alpha$-parabolic Bergman space of order $p$. As in the Hardy case, $b_{1 / 2}^{p}$ coincides with the usual harmonic Bergman space on the upper half space. Let $\mu$ be a positive Borel measure on $\mathbb{R}_{+}^{n+1}$ and $\tau$ be a positive number. We say that $\mu$ is a $\tau$-Carleson measure if there exists a positive constant $C$ such that 


$$
\mu\left(Q^{(\alpha)}(x, t)\right) \leq C t^{(n / 2 \alpha+1) \tau}
$$

holds for all $(x, t) \in \mathbb{R}_{+}^{n+1}$, where

$$
\begin{aligned}
& Q^{(\alpha)}(x, t):=\left\{\left(y_{1}, y_{2}, \ldots, y_{n}, s\right) \in \mathbb{R}_{+}^{n+1} \mid t \leq s \leq 2 t\right. \\
&\left.\left|y_{i}-x_{i}\right| \leq t^{1 / 2 \alpha} / 2, i=1,2, \ldots, n\right\} .
\end{aligned}
$$

Carleson inequalities on parabolic Bergman spaces are studied in [NSY1]: Let $1 \leq p \leq q<\infty$ and $\mu$ be a positive Borel measure on $\mathbb{R}_{+}^{n+1}$. Then $\mu$ is a $q / p$-Carleson measure if and only if there exists a positive constant $C$ such that the inequality

$$
\|u\|_{L^{q}\left(\mathbb{R}_{+}^{n+1}, d \mu\right)} \leq C\|u\|_{L^{p}\left(\mathbb{R}_{+}^{n+1}\right)}
$$

holds for all $u \in b_{\alpha}^{p}$.

We have the following proposition.

Proposition 2 Let $\mu$ be a positive Borel measure on $\mathbb{R}_{+}^{n+1}$. For $\tau>0$, we set $\tau_{b}:=\tau(n / 2 \alpha) /(n / 2 \alpha+1)$. Then

(a) if $\mu$ is a $T_{\tau}$-Carleson measure, then $\mu$ is a $\tau_{b}$-Carleson measure,

(b) if $\tau>1$ and $\mu$ is a $\tau_{b}$-Carleson measure, then $\mu$ is a $T_{\tau}$-Carleson measure.

Proof. Since $Q^{(\alpha)}(x, t) \subset T^{(\alpha)}\left(x,\left((n / 4)^{\alpha}+2\right) t\right)$ for $(x, t) \in \mathbb{R}_{+}^{n+1}$, we have

$$
\begin{aligned}
\mu\left(Q^{(\alpha)}(x, t)\right) & \leq \mu\left(T^{(\alpha)}\left(x,\left(\left(\frac{n}{4}\right)^{\alpha}+2\right) t\right)\right) \\
& \leq C\left(\left(\frac{n}{4}\right)^{\alpha}+2\right)^{(n / 2 \alpha) \tau} t^{(n / 2 \alpha) \tau} \leq C t^{(n / 2 \alpha+1) \tau_{b}}
\end{aligned}
$$

which shows (a). To show (b), we set

$$
T_{k}:=\left\{(y, s) \in T^{(\alpha)}(x, t) \mid t / 2^{k+1} \leq s \leq t / 2^{k}\right\}
$$

for $k=0,1,2, \ldots$ and take a natural number $c(k)$ such that $\left(2^{(k+1 / 2 \alpha+1)}+\right.$ $1)^{n} \leq c(k) \leq 2^{((k+1) / 2 \alpha+2) n}$. Since 


$$
\left[\frac{\left(t-t / 2^{k+1}\right)^{1 / 2 \alpha}}{\left(t / 2^{k+1}\right)^{1 / 2 \alpha}}\right]+1 \leq 2^{((k+1) / 2 \alpha+1)}+1
$$

where $[t]$ is the largest integer smaller than or equal to $t$, we can choose $c(k)$ points $\left\{\left(x_{k, i}, t / 2^{k+1}\right)\right\}$ in $T_{k}$ such that $T_{k} \subset \bigcup_{i=1}^{c(k)} Q^{(\alpha)}\left(x_{k, i}, t / 2^{k+1}\right)$. Hence

$$
\begin{aligned}
\mu\left(T^{(\alpha)}(x, t)\right) & \leq \sum_{k=0}^{\infty} \mu\left(T_{k}\right) \leq \sum_{k=0}^{\infty} \sum_{i=1}^{c(k)} \mu\left(Q^{(\alpha)}\left(x_{k, i}, t / 2^{k+1}\right)\right) \\
& \leq \sum_{k=0}^{\infty} \sum_{i=1}^{c(k)} C\left(t / 2^{k+1}\right)^{(n / 2 \alpha+1) \tau_{b}} \\
& \leq C \sum_{k=0}^{\infty} 2^{((k+1) / 2 \alpha+2) n}\left(t / 2^{k+1}\right)^{(n / 2 \alpha+1) \tau_{b}} \\
& \leq C t^{(n / 2 \alpha) \tau} \sum_{k=0}^{\infty} 2^{k\left(n / 2 \alpha-(n / 2 \alpha+1) \tau_{b}\right)} .
\end{aligned}
$$

Since $n / 2 \alpha-(n / 2 \alpha+1) \tau_{b}=(n / 2 \alpha)(1-\tau)<0, \mu$ is a $T_{\tau}$-Carleson measure.

Theorem 1 and Proposition 2 give us the following corollary.

Corollary $11<p \leq q<\infty$ and let $1 \leq p^{\prime} \leq q^{\prime}<\infty$. Suppose that $(n / 2 \alpha)(q / p)=(n / 2 \alpha+1)\left(q^{\prime} / p^{\prime}\right)$ holds. Then for every positive Borel measure $\mu$ on $\mathbb{R}_{+}^{n+1}$, there exist positive constants $C$ and $C^{\prime}$ such that $\|u\|_{L^{q}\left(\mathbb{R}_{+}^{n+1}, d \mu\right)} \leq C\|u\|_{h_{\alpha}^{p}}$ holds for all $u \in h_{\alpha}^{p}$ if and only if $\|u\|_{L^{q^{\prime}}\left(\mathbb{R}_{+}^{n+1}, d \mu\right)} \leq C^{\prime}\|u\|_{L^{p^{\prime}}\left(\mathbb{R}_{+}^{n+1}\right)}$ holds for all $u \in b_{\alpha}^{p^{\prime}}$.

Acknowledgment The authors would like to thank Professor Yoshihiro Sawano for his comments and discussions.

\section{References}

[L] Luecking D. H., Embedding derivertive of Hardy spaces into Lebesgue spaces. Proc. London Math. Soc. (3) 63 (1991), 595-619.

[NSS1] Nishio M., Shimomura K. and Suzuki N., $\alpha$-parabolic Bergman spaces. Osaka J. of Math. 42 (2005), 133-162. 
[NSS2] Nishio M., Shimomura K. and Suzuki N., L ${ }^{p}$ boundedness of Bergman projections for $\alpha$-parabolic operators. Potential theory in Matsue, 305318, Adv. Stud. Pure Math. 44, Math. Soc. Japan, Tokyo, 2006.

[NSY1] Nishio M., Suzuki N. and Yamada M., Toeplitz operators and Carleson measures on parabolic Bergman spaces. Hokkaido Math. J. 36 (2007), 563-583.

[NSY2] Nishio M., Suzuki N. and Yamada M., Carleson inequalities on parabolic Bergman spaces. Tohoku Math. J. 62 (2010), 269-286.

[S] Stein E. M., Singular integrals and differentiability properties of functions. Princeton Univ. Press, Princeton, New Jersey, 1970.

\author{
Hayato NAKAGAWA \\ Graduate School of Mathematics \\ Nagoya University \\ Chikusa-ku, Nagoya, 466-8602, Japan \\ E-mail: m04026b@math.nagoya-u.ac.jp \\ Noriaki SuzukI \\ Department of Mathematics \\ Meijyo University \\ Tenpaku-ku, Nagoya, 468-8502, Japan \\ E-mail: suzukin@meijo-u.ac.jp
}

